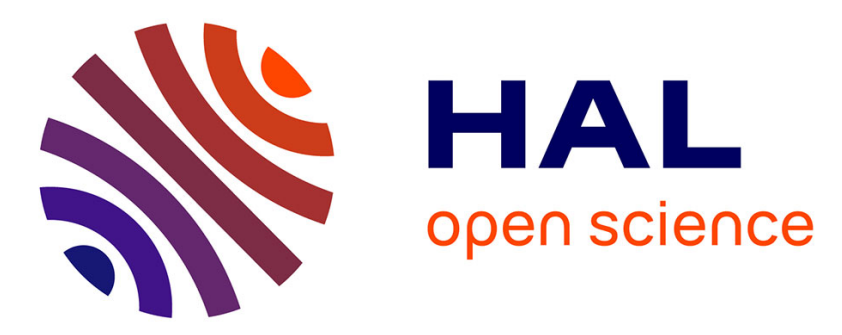

\title{
Comparaison précise de courants périodiques à l'aide de thermistances à chauffage indirect
}

\author{
J.C. Bernengo, M. Hanss, P. Rottembourg
}

\section{To cite this version:}

J.C. Bernengo, M. Hanss, P. Rottembourg. Comparaison précise de courants périodiques à l'aide de thermistances à chauffage indirect. Revue de Physique Appliquée, 1967, 2 (3), pp.184-186. 10.1051/rphysap:0196700203018400 . jpa-00242788

\section{HAL Id: jpa-00242788 https://hal.science/jpa-00242788}

Submitted on 1 Jan 1967

HAL is a multi-disciplinary open access archive for the deposit and dissemination of scientific research documents, whether they are published or not. The documents may come from teaching and research institutions in France or abroad, or from public or private research centers.
L'archive ouverte pluridisciplinaire HAL, est destinée au dépôt et à la diffusion de documents scientifiques de niveau recherche, publiés ou non, émanant des établissements d'enseignement et de recherche français ou étrangers, des laboratoires publics ou privés. 


\title{
GOMPARAISON PRÉGISE DE GOURANTS PÉRIODIQUES A L'AIDE DE THERMISTANGES A GHAUFFAGE INDIREGT
}

\author{
Par J. G. BERNENGO, M. HANSS (1) et P. ROTTEMBOURG, \\ Laboratoire de Biophysique du Muséum National d'Histoire Naturelle, Paris \\ et Laboratoire de Chimie Macromoléculaire de la Faculté des Sciences de Lyon.
}

\begin{abstract}
Résumé. - On décrit un pont équipé de deux thermistances à chauffage indirect qui permet la comparaison précise d'un courant périodique (de forme quelconque) à un courant connu (périodique ou non). Dans les conditions les plus favorables, la sensibilité relative peut atteindre $10^{-5}$, entre 0,1 et $500 \mathrm{~Hz}$. Ce dispositif est encore utilisable à des fréquences beaucoup plus élevées.

Abstract. - A very low frequency thermal transfer unit is described. This device is based on the use of two indirectly-heated thermistors, in two adjacent arms of a Wheatstone bridge. A periodic current (of any shape) can thus be compared to a known current (periodic or D.C.) with a relative sensitivity which may reach, under the best conditions, a few parts in $10^{-5}$ between 0,1 and $500 \mathrm{~Hz}$. The bridge is still usable at much higher frequencies.
\end{abstract}

Dans le dispositif précédemment décrit $[1,2]$ destiné à mesurer la dispersion de la conductivité de solutions ioniques en très basse fréquence, deux tubes thermoélectriques placés en opposition nous ont permis de comparer deux courants alternatifs de valeurs voisines avec une précision relative de l'ordre de $10^{-4}$, entre 1 et $500 \mathrm{~Hz}$.

Afin d'améliorer ce chiffre et surtout d'atteindre de plus basses fréquences, nous avons eu recours à des thermistances à chauffage indirect (T.C.I.), constituées par une thermistance et un filament chauffant, ces deux éléments étant électriquement isolés l'un de l'autre. La résistance de l'élément thermosensible diminue lorsqu'on fait passer un courant alternatif ou continu dans le filament; cette propriété est utilisée dans certains dispositifs de régulation ou de commande à distance du gain d'amplificateurs par T.C.I. [3].

En mesurant la valeur de la thermistance, on peut également déterminer avec précision, et sur une très grande plage de fréquence, l'intensité efficace d'un courant périodique inconnu (non nécessairement sinusoïdal) alimentant la T.C.I. C'est ainsi que H. B. Wood [4] a construit un milliampèremètre qui permet de mesurer la valeur efficace d'un courant périodique avec une précision de $4 \%$ jusqu'à $10 \mathrm{MHz}$. Popov [5] a décrit un montage utilisant une T.C.I. pour comparer à la précision relative de $3 \times 10^{-3}$ un courant alternatif $(20 \mathrm{~Hz}-20 \mathrm{kHz})$ à un courant continu connu. Il nous semble quand même utile de présenter notre pont de comparaison à deux T.C.I., dont la sensibilité relative peut être meil-

(1) Adresse actuelle : Division de Microbiologie du C.R.S.S.A., 108, boulevard Pinel, Lyon (3e). leure que $10^{-4}$, et qui est utilisable à des fréquences aussi basses que $0,1 \mathrm{~Hz}$.

Nous avons essayé les T.C.I. suivantes :

- type B 23 (chauffage $100 \Omega$ ) de STG (éléments sous vide, en ampoule de verre, résistance nominale 2 à $3 \mathrm{k} \Omega$ ),

- type B8 32015 P 3K3 (chauffage $50 \Omega$ ) de la Radiotechnique.

En raison de leur plus grande constante de temps (25 $\mathrm{s}$ au lieu de $2 \mathrm{~s}$ ), nous avons adopté les thermistances STC pour les basses fréquences, réservant le deuxième type à des applications en plus haute fréquence (une faible constante de temps permet alors de détecter des modulations rapides du courant).

Les thermistances étant très sensibles aux fluctuations de la température ambiante, la détermination directe (ou par pont de Wheatstone) de leur valeur ne permet pas des mesures de courant très précises. C'est pourquoi il est préférable d'adopter une méthode différentielle en utilisant dans les branches adjacentes d'un pont deux T.G.I. identiques, leurs enveloppes étant en court-circuit thermique.

Un tel montage peut être employé de deux façons :

- soit pour comparer directement deux courants $i_{1}$ et $i_{2}$ de valeurs voisines, traversant les filaments chauffants des deux T.C.I. [4],

- soit pour obtenir une mesure absolue de la valeur efficace d'un courant périodique $i$ (de forme quelconque) qui chauffe une des deux T.G.I. On équilibre alors le pont en ajustant l'intensité d'un courant de tarage, $i_{0}$, alimentant la deuxième T.G.I. Il est inutile de connaître la valeur de $i_{0}$, mais ce courant doit être stable une fois l'équilibre atteint. On substitue ensuite à $i$ un courant continu (dont on 
peut mesurer l'intensité avec une grande précision) que l'on fait varier de manière à retrouver l'équilibre : sa valeur est alors égale à la valeur efficace de $i$.

Pour ces deux types d'utilisation, la sensibilité et la précision ultimes que l'on peut espérer sont limitées par la stabilité du pont pendant la durée de la comparaison ou de la substitution. Cette stabilité a été étudiée à l'aide du montage de la figure 1 . Le pont est formé

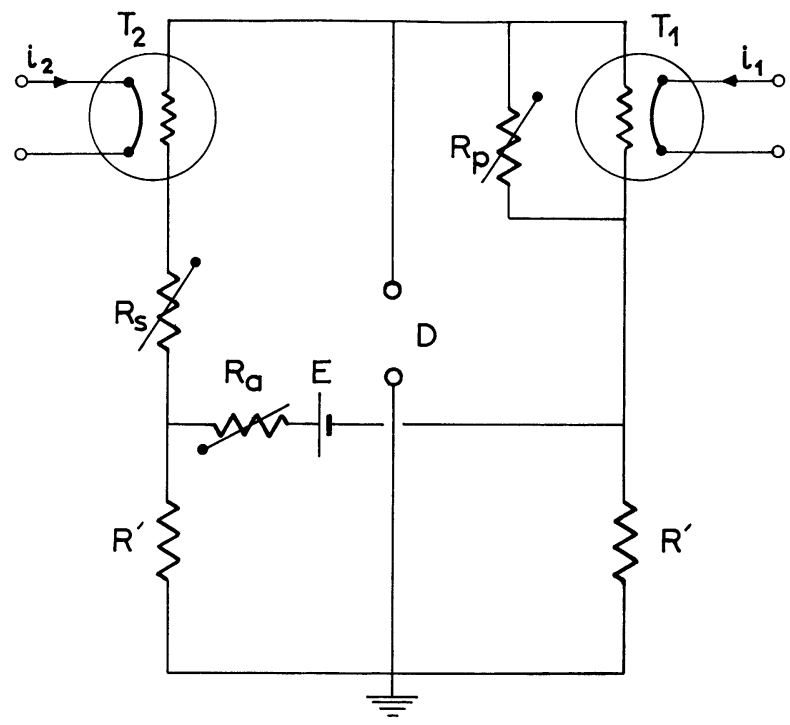

FIG. 1. - Schéma du pont de comparaison à deux T.C.I.

des thermistances $T_{1}$ et $T_{2}$, des résistances $R^{\prime}$ et des résistances réglables $R_{\mathrm{s}}$ et $R_{\mathrm{p}}$. Il est alimenté par un élément de pile au mercure, en série avec la résistance $R_{\mathrm{a}}$, dont le rôle est de limiter le courant d'alimentation à un niveau suffisamment faible pour ne pas modifier la valeur des thermistances en les chauffant directement. Les tensions de déséquilibre $\Delta \mathrm{V}$ sont enregistrées à l'aide d'un appareil Graphispot précédé d'un Amplispot « Sefram ». Le pont ayant été équilibré au préalable, toute variation de la différence

$$
\Delta i=i_{1}-i_{2}
$$

se traduira par une déviation, que l'on pourra ensuite convertir en $\Delta i$ après étalonnage.

Nous avons construit la courbe $R=f(i) \quad(R=$ résistance de la thermistance) pour un élément STG (fig. 2). La sensibilité est mesurée par une « pente de conversion » $\mathrm{d} R / \mathrm{d} i$, maximale au point d'inflexion. En pratique, on voit donc qu'avec ce type de T.G.I. il faudra choisir un point de fonctionnement compris entre 2 et $10 \mathrm{~mA}$ pour obtenir la meilleure sensibilité.

La réjection du mode commun n'est pas infinie car les deux T.G.I. ne sont pas absolument identiques. Néanmoins, elle peut être très améliorée en agissant sur $R_{\mathrm{s}}$ et $R_{\mathrm{p}}$. En effet, soit deux T.C.I. de caractéristiques différentes, alimentées par le même courant $i$; à l'aide de $R_{\mathrm{s}}$ (placée en série avec la thermistance la

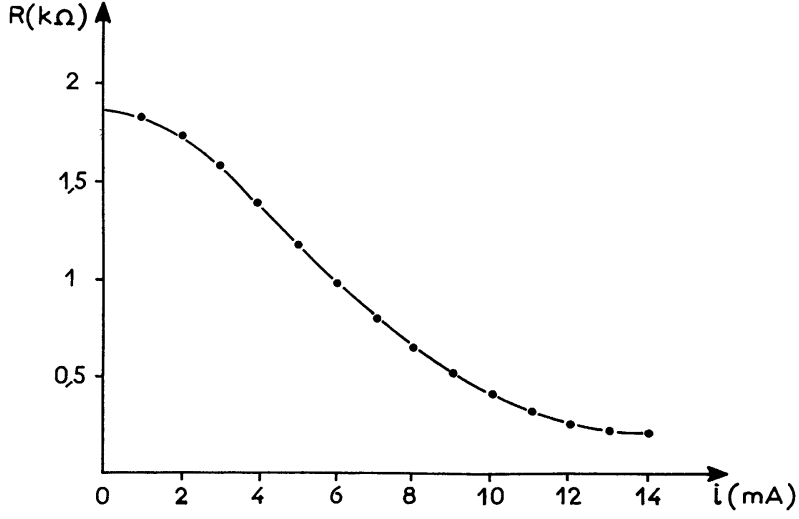

FIG. 2. - Résistance d'une T.C.I. en fonction du courant de chauffage.

moins résistante), on peut faire coïncider les deux points des courbes $R=f(i)$ correspondant à $i$. Ensuite, en modifiant $R_{\mathrm{p}}$, nous pourrons égaliser les pentes des courbes pour le point de fonctionnement choisi. Comme ces réglages ne sont pas indépendants, il faut procéder par tâtonnements. Finalement, les deux T.C.I. se comporteront comme ayant exactement les mêmes caractéristiques dynamiques pour la valeur $i$.

La « réjection du mode commun thermique » peut, elle aussi, être insuffisante parce que les impédances de transfert thermiques entre le filament et la thermistance des T.C.I. diffèrent entre elles, mais il n'est pas possible d'agir sur cette dissymétrie. Il est préférable d'utiliser deux T.C.I. aussi semblables que possible si l'on veut éviter de réajuster $R_{\mathrm{s}}$ et $R_{\mathrm{p}}$ chaque fois que l'on choisit un point de fonctionnement différent. G'est pourquoi nous avons sélectionné quelques paires de T.C.I. de caractéristiques très voisines dans la gamme de courant à mesurer (nous avons constaté en effet une grande dispersion des caractéristiques).
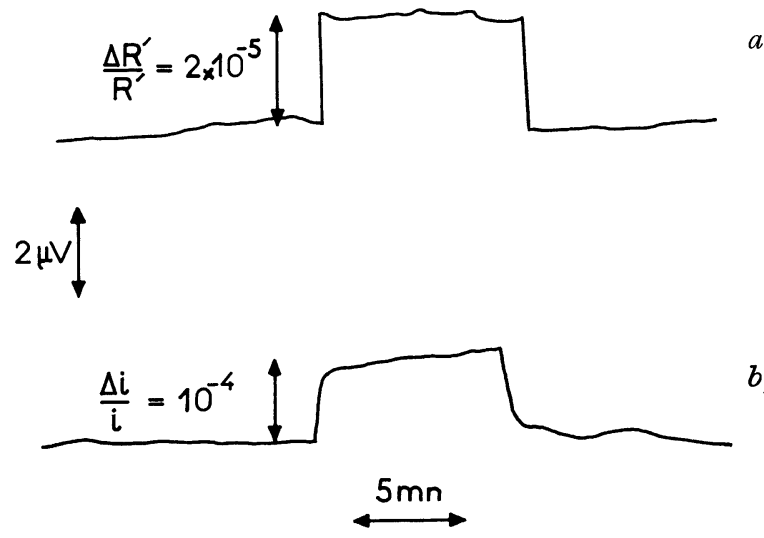

FIG. 3.

a) Signal de déséquilibre du pont pour une variation relative de $2 \times 10^{-5}$ de $R^{\prime}$

b) Signal obtenu pour une variation relative de $10^{-4}$ du courant de chauffage de $T_{1}\left(i_{1} \simeq 5 \mathrm{~mA}\right)$. 
La figure 3 montre que la même tension de déséquilibre apparait, soit pour une variation

$$
\Delta R^{\prime} \mid R^{\prime}=2 \times 10^{-5}
$$

de la résistance d'une branche du pont, soit pour une variation de courant $\Delta i / i=10^{-4}$ dans une des deux T.C.I. : le facteur de conversion $\frac{\Delta R^{\prime}}{R^{\prime}} / \frac{\Delta i}{i}$ est donc égal à 0,2 environ. On remarque également que le bruit correspond à une variation relative de courant de $\pm 2 \times 10^{-5}$; ce chiffre détermine donc l'ordre de grandeur de la sensibilité du dispositif.

Le pont peut être employé sans précautions particulières à des fréquences aussi basses que $1 \mathrm{~Hz}$, en raison de la grande constante de temps des T.C.I. et de leur réjection de mode commun thermique; en effet, cette dernière caractéristique tend à supprimer l'ondulation résiduelle lorsque $i_{1}$ et $i_{2}$ sont en phase. Pour descendre plus bas en fréquence, il devient nécessaire d'employer des T.C.I. appariées en constante de temps (fig. 4). De plus, il est possible d'équilibrer le pont malgré un certain taux d'ondulation, si bien que le montage précédent permet de comparer la valeur efficace de deux courants périodiques avec une précision relative meilleure que $10^{-4}$, entre 0,1 et $500 \mathrm{~Hz}$.

Nous avons appliqué par ailleurs le montage précédent équipé de deux T.G.I. de la Radiotechnique, à l'enregistrement de variations lentes de la différence entre deux courants alternatifs à $200 \mathrm{kHz}$. Les résultats concernant le bruit et la dérive (inférieure à $10^{-4} \mathrm{du}$ courant traversant la T.G.I.) étaient comparables. Le facteur de conversion de la T.C.I. n'est réduit que de moitié lorsque la fréquence atteint $40 \mathrm{MHz}$.

En conclusion, le pont de thermistances à chauffage indirect que nous utilisons permet de comparer avec

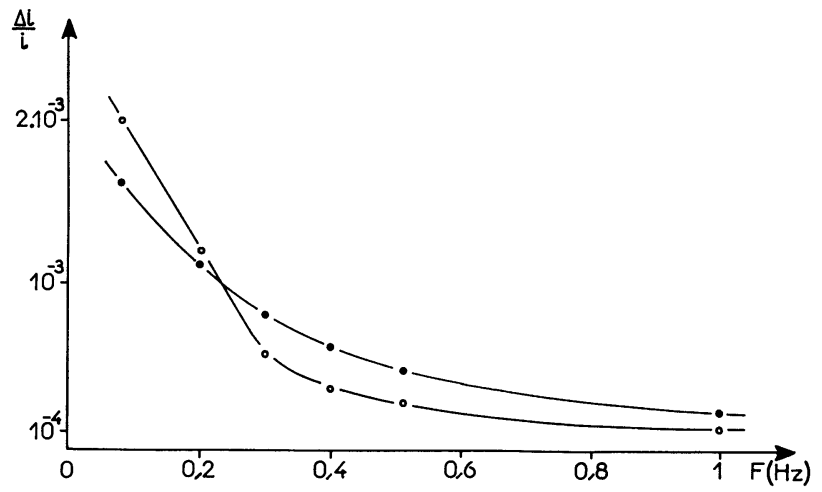

FIG. 4. - Ondulation résiduelle du pont en fonction de la fréquence (ramenée à une variation relative $\Delta i / i$ de l'un des courants de chauffage) pour deux paires de T.C.I. différentes.

une très bonne sensibilité, et à des fréquences aussi basses que $0,1 \mathrm{~Hz}$, la valeur efficace de deux courants périodiques, complètement isolés l'un de l'autre et de la masse. Il offre également la possibilité de mesurer à la précision relative de $4 \times 10^{-5}$ la valeur efficace d'un courant périodique de forme quelconque auquel on substitue un courant continu connu. Toutefois, ces mesures sont limitées à des valeurs comprises approximativement entre 1 et $10 \mathrm{~mA}$; il faut de plus choisir deux éléments appariés et en ajuster les caractéristiques. Malgré ces inconvénients, il nous semble que notre dispositif peut rendre service dans un certain nombre d'applications, tant en raison de sa grande sensibilité que de son indépendance vis-à-vis de la fréquence.

Manuscrit reçu le 4 février 1967.

\section{BIBLIOGRAPHIE}

[1] Hanss (M.), Thèse, Paris, 1965.

[2] Hanss (M.), Guermonprez (R.), J. Chim. Physique, 1966, 63, 663.
[3] Hutcheon (I. C.), in "Tunnel-Diodes and semiconductors " (J. M. Carroll, éd.), McGraw-Hill, 1963.

[4] Wood (H. B.), J. Sri. Instr., 1954, 31, 124.

[5] Popov (V. S.), Elektrichestvo SSSR, 1963, 7, 76. 